\title{
A Monte-Carlo Benchmark of TRIPOLI-4® and MCNP on ITER neutronics
}

\author{
David Blanchet ${ }^{1, a}$, Yannick Pénéliau¹, Romain Eschbach¹, Bruno Fontaine ${ }^{1}$, Bruno Cantone ${ }^{2}$, Marc Ferlet ${ }^{2}$, Eric Gauthier², \\ Christophe Guillon ${ }^{2}$, Laurent Letellier ${ }^{2}$, Maxime Proust ${ }^{2}$, Fernando Mota ${ }^{3}$, Iole Palermo ${ }^{3}$, Luis Rios ${ }^{3}$, Frédéric Le Guern ${ }^{4}$, \\ Martin Kocan ${ }^{5}$, Roger Reichle ${ }^{5}$
}

${ }^{1}$ CEA,DEN/DER, Cadarache, 13108 Saint-Paul-lez-Durance, France

${ }^{2}$ CEA, IRFM, Cadarache, 13108 Saint-Paul-lez-Durance, France

${ }^{3}$ CIEMAT, Avda.Complutense, 40, Madrid 28040, Spain

${ }^{4}$ F4E, Josep Pla 2, Torres Diagonal Litoral B3, 08019 Barcelona, Spain

5ITER Organization, Route de Vinon sur Verdon, CS 90 046, 13067 Saint-Paul-lez-Durance, France

\begin{abstract}
Radiation protection and shielding studies are often based on the extensive use of 3D Monte-Carlo neutron and photon transport simulations. ITER organization hence recommends the use of MCNP-5 code (version 1.60), in association with the FENDL-2.1 neutron cross section data library, specifically dedicated to fusion applications. The MCNP reference model of the ITER tokamak, the ' $\mathrm{C}$-lite', is being continuously developed and improved. This article proposes to develop an alternative model, equivalent to the 'C-lite', but for the Monte-Carlo code TRIPOLI-

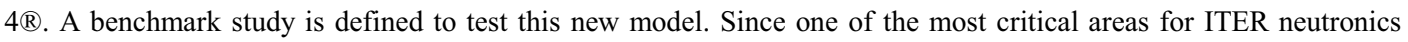
analysis concerns the assessment of radiation levels and Shutdown Dose Rates (SDDR) behind the Equatorial Port Plugs (EPP), the benchmark is conducted to compare the neutron flux through the EPP. This problem is quite challenging with regard to the complex geometry and considering the important neutron flux attenuation ranging from $10^{14}$ down to $10^{8} \mathrm{n} \cdot \mathrm{cm}^{-2} \cdot \mathrm{s}^{-1}$. Such code-to-code comparison provides independent validation of the Monte-Carlo simulations, improving the confidence in neutronic results.
\end{abstract}

\section{Introduction}

One of the most critical issues for ITER neutronics analysis concerns the assessment of radiation levels behind the Equatorial Port Plugs. The Port Plugs are water-cooled stainless steel structures designed to host a wide variety of diagnostic systems. Among them, the Equatorial Visible/Infra-Red Wide Angle Viewing System (WAVS) [1] plays a key role in machine protection by providing on-line monitoring of the surface temperature of plasma facing components, in order to avoid excessive heating. In addition to support diagnostics, the Port Plugs shall provide sufficient radiation shielding to attenuate the propagation of the intense, high-energy plasma-neutron flux. However, the Port Plug itself in the Port and the diagnostics create feedthroughs and streaming gaps, allowing the neutron flux to penetrate deeply into the Port Plug structures. This neutron irradiation could lead to significant activation of structural materials, with subsequent decay gamma emissions, contributing to the shutdown dose rate.

In order to limit the occupational radiation exposure during maintenance, the shutdown dose rate behind the Port Plug, in the Port Interspace area (Figure 1), should not exceed $100 \mu \mathrm{Sv} \cdot \mathrm{h}^{-1}$ after 12 days shutdown. Actually, recent studies [2] have shown that this project criterion is particularly challenging. Improvements are sought in the

\footnotetext{
a Corresponding author: david.blanchet@cea.fr
}

design of the shielding of the radiation streaming through the gaps and penetrations (adopting optical labyrinths) and in the choice of materials (use of boron carbide as neutron absorber). High density materials should be avoided because of severe constraints on weight limits. Moreover, the problem cannot be solved locally without considering all contributions, because of the cross-talk interferences (contributions from neighbouring components), mainly from the Lower Ports. Hence, there is a need to consider complete models with a lot of details included and to develop them in a modular and flexible approach allowing regular updates during the design phase. The convergence of Monte-Carlo simulations on these large-scale models requires the use of biaising techniques for variance reduction. This last point highlights the importance of cross-checking the simulations. There is an interest in proposing alternative models for independent validations that will improve the confidence in the neutronic results. For this reason, the present study describes the development of an equivalent 'C-lite' model but for the Monte-Carlo code TRIPOLI-4® [3] as explained in Section 2. A benchmark study is also defined to test the TRIPOLI-4® ITER model as detailed in Section 3. The results of such code-to-code comparison are provided in Section 4. 


\section{Development of the ITER C-lite TRIPOLI-4® model}

ITER organization recommends the use of MCNP-5 [4] code (version 1.60), in association with the FENDL-2.1 [5] neutron cross section data library, specifically dedicated to fusion applications. The MCNP reference model of the ITER tokamak, known as the 'C-lite', is a representation of the machine in a $40^{\circ}$ torus sector, with specular reflections at the $\pm 20^{\circ}$ boundaries. The C-lite model integrates generic descriptions of the components, but depending on the level of interest, it can also incorporate very detailed and sophisticated descriptions of the components under study. In the present study, we consider a MCNP C-lite model composed of about 10 ’000 volumes.

\subsection{Methodology for Geometry Creation}

The TRIPOLI-4® model is created, component-bycomponent in order to preserve the original structure of the C-lite based on Universes. The MCNP Universes can be translated in TRIPOLI-4® by using fictitious volumes. These fictitious volumes can be seen as containers, to be filled with detailed component descriptions using volume intersection operator. This way of creating the geometry ensures the required flexibility for regular update of a particular component if it is needed or if a refined model is available. This is the case of the Diagnostic Generic Equatorial Port Plug (DGEPP) models which are of particular interest in the present study. The length of the EPP represented in figure 1 is about $\sim 3$ meters.
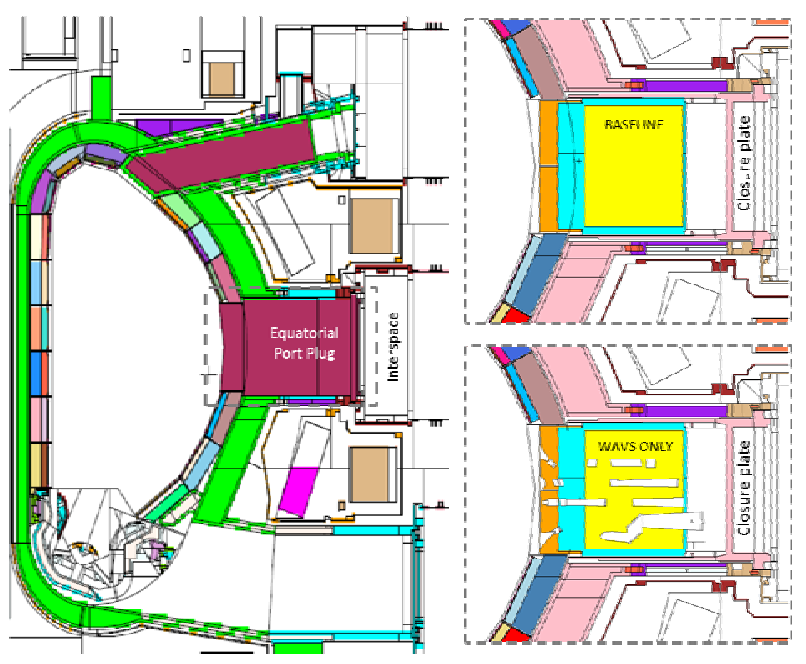

Figure 1. RZ (poloidal) view of the generic EPP in MCNP geometry (left); detailed views of the "baseline" (top right) and "WAVS only" (bottom right) DGEPP models.

Among the various diagnostics to be housed by the EPP, this study only focuses on the WAVS diagnostics. The optical system of the WAVS diagnostics is represented by 4 lines-of-sight (respectively for the Upper, Left, Right and Divertor views) creating apertures and feedthroughs in the Diagnostic Shield Module (DSM) structure. In order to avoid direct streaming corridors for neutrons, dogleg configurations are highly recommended; the number and the position of doglegs are still under optimization. The optical paths of the WAVS system are illustrated in Figure 2.

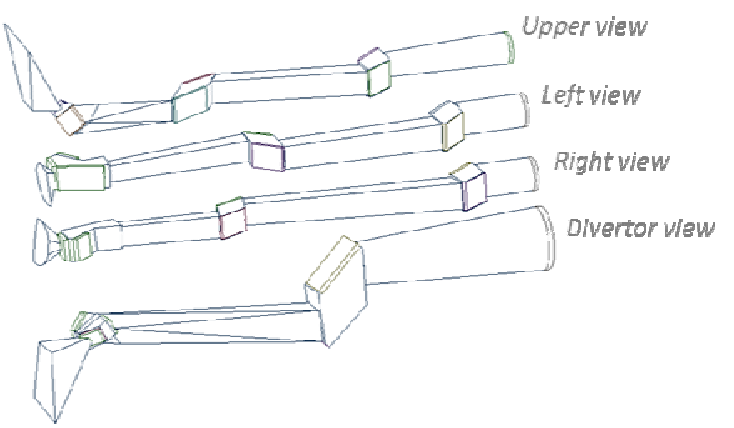

Figure 2. CAD model of the WAVS optical paths (with dogleg configurations) through the EPP (length $\sim 3 \mathrm{~m}$, diameter $<16$ $\mathrm{cm})$.

The CAD models of the detailed components are first simplified using SpaceClaim Engineer 2015 CAD tool [6]. This simplification basically consists in: removing all unnecessary elements (which do not influence the transport analyses of the high energy neutrons) and preserving only the most important ones; substituting the sp-lines with surfaces understandable by the code (i.e. second-degree surfaces and toroidal fourth-degree surfaces); splitting the large and complex components into simple ones, and finally filling the little voids because all the space must be defined to ensure particle transport doesn't stop in a void medium. Besides, MonteCarlo codes require void cell descriptions, which are generally lacking in the CAD models, hence complementary cells have to be defined. This can be done by modifying the original CAD geometry, either directly with SpaceClaim or later on with MCAM.

During this complex simplification process, care should be taken to preserve mass balance when the volumes are modified: this is done by adjusting material densities. An illustration of this simplification process is proposed in Figure 3.
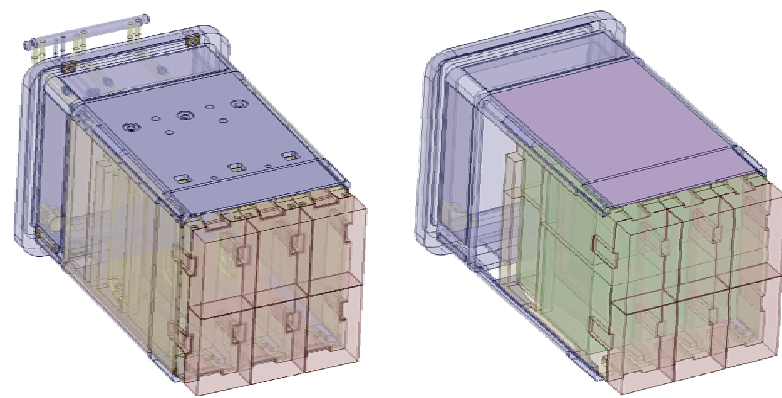

Figure 3. Simplification of the DGEPP CAD model with SpaceClaim (on left: the original design, on right: the simplified design).

Once simplified, the CAD geometry can be converted to MCNP-5 or TRIPOLI-4® geometry using MCAM (Professional Version 5.2) software [7]. The created Monte-Carlo geometries are finally included into the $\mathrm{C}$ lite model.

In Figure 4, part (b) (c) and (d) represent the successive steps in the TRIPOLI-4® geometry creation. These illustrations were generated using the embedded 
plot utility named T4G [8]. This tool allows considerably fast display and navigation into the geometry.

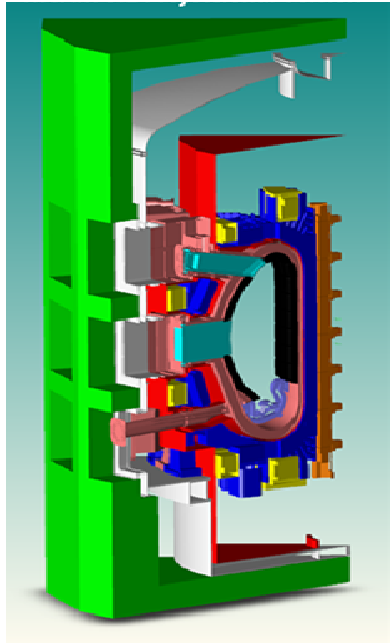

(a)

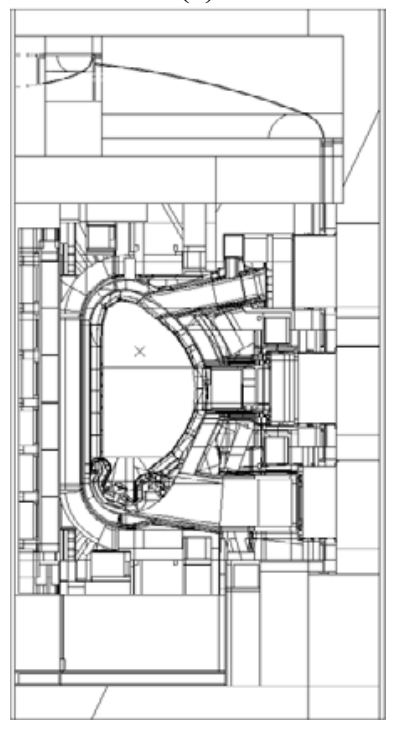

(c)

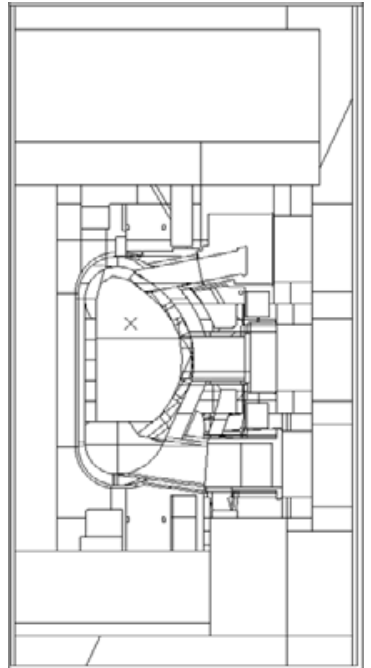

(b)

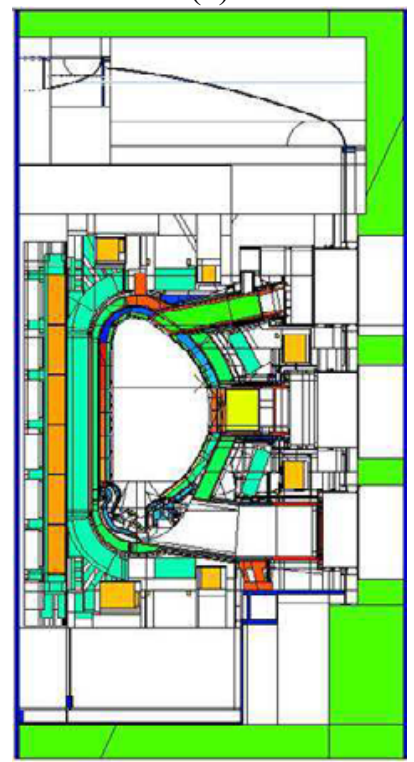

(d)
Figure 4. Development of the ITER ' $\mathrm{C}$-lite $\mathrm{V} 1$ ' model in TRIPOLI-4®. (a) Original CAD model in step format. (b) Definition of fictitious volumes representing Universes in TRIPOLI-4®. (c) Definition of detailed Components. (d) Geometry to material association.

The TRIPOLI-4® geometry is tested by counting lost particles when all cells are filled with void material. It is mandatory to get zero lost particles in simulations of at least $10^{7}$ source particle histories run. Another interesting geometrical test consists in the stochastic volume estimation by ray tracing performed by setting a uniform neutron source distribution inward-directed, on a plane surface, voiding out all materials in the problem and setting all nonzero importances to one. In this way the calculation of the neutron flux tallies (using the tracklength estimator, F4 in MCNP and TRACK in TRIPOLI$4 \AA)$ provide Monte-Carlo estimates of all cell volumes. These checks were successfully done in both MCNP-5 and TRIPOLI-4® models showing a perfect agreement of the volumes calculated by the two codes when they are compared with the direct CAD volumes. Following this analysis, the volumes and the mass balance of DGEPP cells are deduced and pointed out in Table 1.

Table 1. Volume and mass Balance in dgepp-0-wavs model (estimations by TRIPOLI-4®).

\begin{tabular}{|c|c|c|c|}
\hline & Density & Volumes & Masse \\
\hline Material & t. $\mathrm{m}^{-3}$ & $\mathbf{m}^{3}$ & $\mathbf{t}$ \\
\hline SS316L(N) & 7,93 & 2,12 & 16,782 \\
\hline SS316L(N)+10\%H2O & 7,19 & 0,81 & 5,845 \\
\hline SS316L(N)+5\%H2O & 7,58 & 2,48 & 18,833 \\
\hline B4C & 1,89 & 3,03 & 5,731 \\
\hline TOTAL DGEPP & 5,59 & 8,45 & 47,191 \\
\hline
\end{tabular}

Considering fully shielded DSM is a rather conservative assumption for the total EPP weight estimate which is already exceeding the 45 tons limit imposed by the project. According to the above table, the shielding material in the EPP could not exceed $\sim 3.5$ tons. It is clear that high density materials should be avoided.

\subsection{Verification of the D-T neutron source}

The ITER standard neutron source is defined by the nominal inductive operation scenario, which assumes a deuterium-tritium plasma pulse with a total fusion power of $500 \mathrm{MW}$. The energy distribution of the $14 \mathrm{MeV}$ neutrons emitted by D-T reactions is described by the built-in Gaussian fusion energy distribution, centred at $\mathrm{E}$ $=14.0791 \mathrm{MeV}$, with a spread of $\sigma=0.53415 \mathrm{MeV}$. The total neutron emission rate is normalized to $1.9718 \cdot 10^{19}$ neutrons per second. Figure 5 is an illustration of the toroidally-averaged poloidal distribution of the neutron source, as sampled in analog (unbiased) simulations by the Monte-Carlo TRIPOLI-4® code.
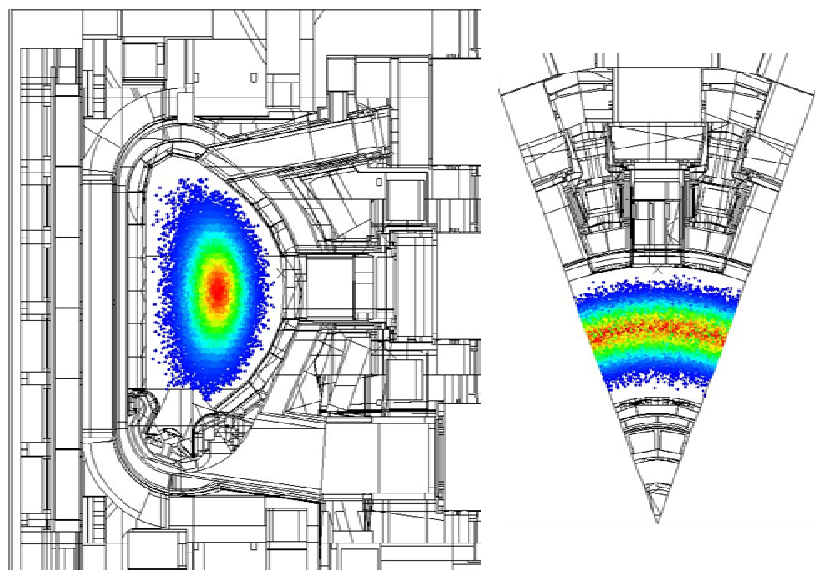

Figure 5. Source Density Sampling by TRIPOLI-4®.

\subsection{Assessment of Variance Reduction Techniques}

Variance-reduction techniques are used in both MCNP-5 and TRIPOLI-4® to improve statistics (Weight Windows 
in MCNP, Exponential Transform in TRIPOLI-4® [9] [10]).

In MCNP, the automatic weight windows generator is used on fictitious geometry with cells having artificially reduced material densities (by a factor 0.1). This technique is widely recognized to easier the production of importance maps on large geometries, especially when there are strong neutron flux attenuations. Neutron importance maps are generated on a rather broad energy mesh in 3 groups (defined with boundaries at $10^{-11}, 10^{-3}$, 0.1 and $20 \mathrm{MeV}$ ), which is efficient for hard neutron spectra. An irregular spatial mesh is defined to match the various components (mesh sizes might be varying from $10 \mathrm{~cm}$ up to few meters). The resulting maps are plotted in Figure 6, where blue zones are standing for high importance regions. These maps were obtained by specifying a target detector at the rear of the Equatorial Port Plug. They were improved by several iterations in a so-called learning approach.
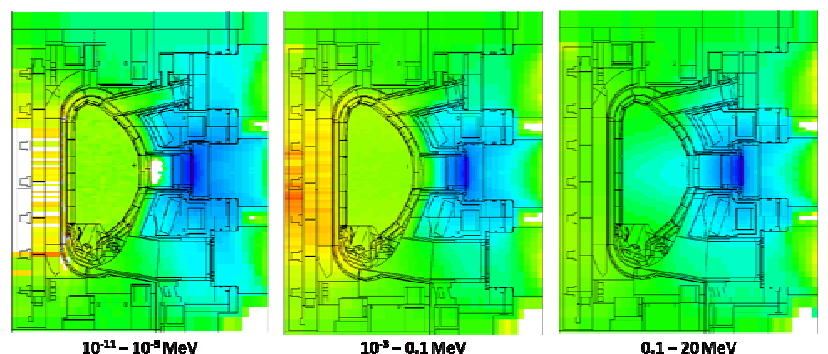

Figure 6. Weight Windows map for MCNP.

In a first attempt, the same spatial and energy meshes are used for the automatic biasing scheme implemented in TRIPOLI-4®. As opposed to the user-friendly generation of weight windows in MCNP which relies on brute force to be efficient (thanks to the automatic learning approach), the "automatic" ponderation in TRIPOLI-4®, requires much efforts and expertise in preliminary tuning to be efficient.

The preliminary importance maps generated by TRIPOLI-4® using a cylindrical detector are illustrated in Figure 7. These importance maps are not yet very satisfactory and would require further optimization. This will be investigated in a future work.
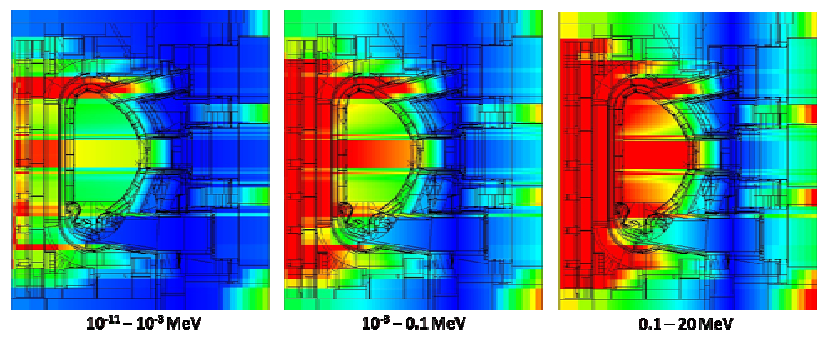

Figure 7. Importance maps obtained with automatic variance reduction technique in TRIPOLI-4®.

The efficiency of the variance reduction is assessed by comparison with analog simulations (i.e. simulations not using any variance reduction, except for the implicit capture). General characteristics of these simulations are reported in Table 2 . The improvement induced by the variance reduction technique is measured by a statistical indicator denoted FoM and defined as the Figure of Merit:

$$
F o M=\frac{1}{R^{2} \cdot T}
$$

Where $\mathrm{R}$ is the relative standard deviation and $\mathrm{T}$ is the computer running time. Results having the highest FoM are preferred. According to Table 2, weight windows are especially improving the statistical convergence of neutron flux results at the target location.

Table 2. Example of statistical indicators (figure of merit) associated to neutron flux obtained either in analog simulations or in simulations using variance reduction techniques. These computations were run on parallel machines at the CEA supercomputing center, using 256 cores from the Curie nodes (Intel ${ }^{\circledR}$ Nehalem-Ex X7560 at $2.26 \mathrm{GHz}$ ).

\begin{tabular}{|c|c|c|c|}
\hline & \multicolumn{2}{|c|}{ Analog Simulation } & Variance Reduct $^{\circ}$ \\
\hline & T-4 & MCNP & MCNP \\
\hline Time (h) & 501 & 468 & 302 \\
\hline Particles (\#) & $10^{9}$ & $10^{9}$ & $5 \cdot 10^{9}$ \\
\hline Std-Dev(\%) & $2.0 \%$ & $1.6 \%$ & $0.7 \%$ \\
\hline FoM & 5.0 & 8.9 & 67.6 \\
\hline
\end{tabular}

Such a comparison with analog simulations is not only meant to measure the performance of the variance reduction, but it provides also an additional check on the absence of deviation introduced by the biasing techniques. Values obtained with variance reduction were found in good agreement with those coming from analog simulations according to their statistical error uncertainty.

\section{Description of the benchmark study}

This study is conducted to assess the radiation level at the rear of the EPP, the neutron fluxes being calculated at the Closure Plate (CP) and at the surrounding Closure Flange (CF) of the EPP. The activation of the closure plate is one of the most important contributions to the shutdown dose rate in the Interspace. For this reason, the calculation of the neutron flux at the closure plate can be considered as a preliminary step before the determination of shutdown dose rates in the Interspace.

The following two DGEPP models are being considered for the benchmark study:

- a model denoted as "dgepp-0" representing the initial design;

- a model denoted as "dgepp-1" representing the optimized design, with improved neutron shielding features.

The optimized design ("dgepp-1") incorporates some neutron labyrinths in the surrounding gaps between the Port Plug and the Vessel; and some neutron stoppers made of natural boron carbide in the inter-drawer gaps. These neutron shielding features are well-illustrated in Figure 7. In this figure, it can also be observed that the DGEPP is divided into 3 drawers filled with Diagnostic 
Shield Modules (DSM) made of powdered natural $\mathrm{B}_{4} \mathrm{C}$ at $1.89 \mathrm{~g} / \mathrm{cm}^{3}$ density. Two configurations are under study:

- The "baseline" configuration, which does not house any diagnostic inside, represents a fully dense EPP, having 3 drawers completely filled by the DSM.

- The "wavs" configuration represents the EPP with the 4 optical paths of the WAVS diagnostics included in one of the 3 drawers (the other ones being full of shielding material).

The study measures the impact that the WAVS diagnostics have on the flux level at the back-end of the EPP. In this approach, all possible contributions from other diagnostics are voluntary ignored. Some illustrations of these four models are proposed in the Figure 8.

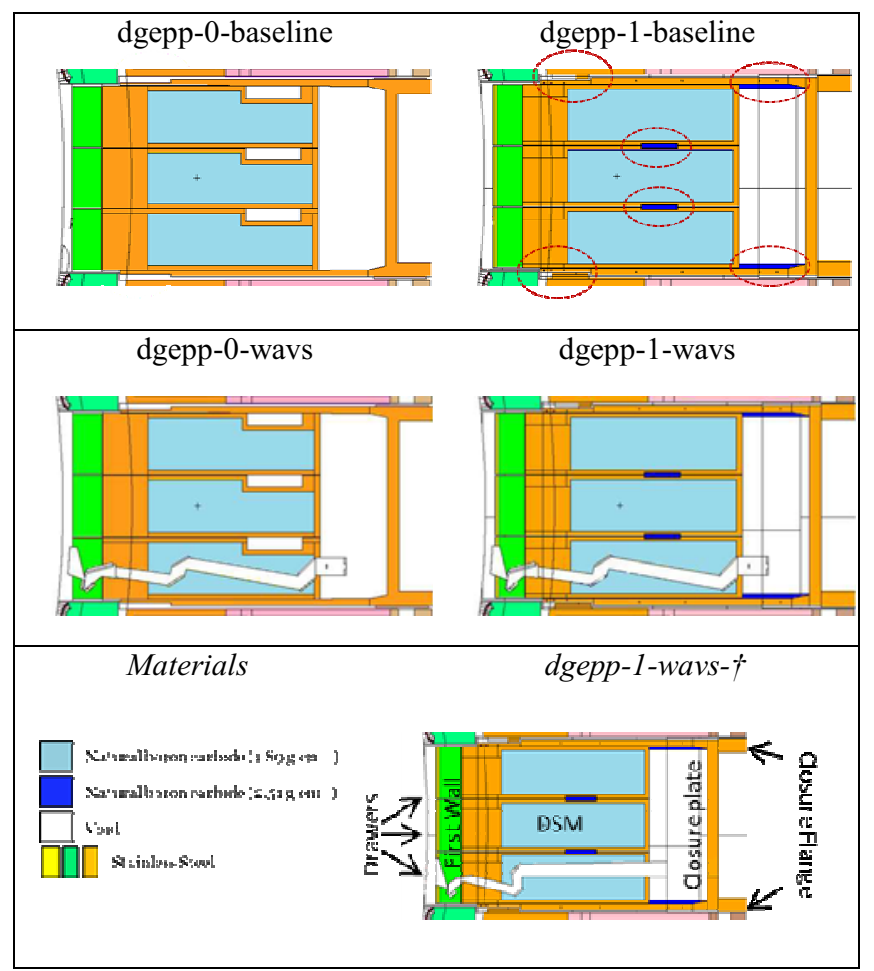

Figure 8. Benchmark cases. (†Alternative optical paths defined by only 1 dogleg instead of 2 ).

There are on-going studies to investigate alternative designs for the optical paths, by looking at the impact of adopting one dogleg instead of two, as illustrated in the additional configuration denoted as "dgepp-1-wavs- $\dagger$ " in Figure 8. However this case is presented only for illustration purpose and will not be considered in the rest of the study.

\section{Results}

\subsection{Neutron Flux at the Equatorial Port Plug}

Neutron spectra are calculated at the closure plate by the use of MCNP-5 and TRIPOLI-4®. Some of these results are depicted in Figure 9. The energy-integrated neutron flux values are reported in Table 3. All comparisons between MCNP-5 and TRIPOLI-4 $\AA$ have revealed an excellent agreement; according to Table 3 for instance, there is no difference at all between the results obtained by the two codes in consideration of the statistical errors.

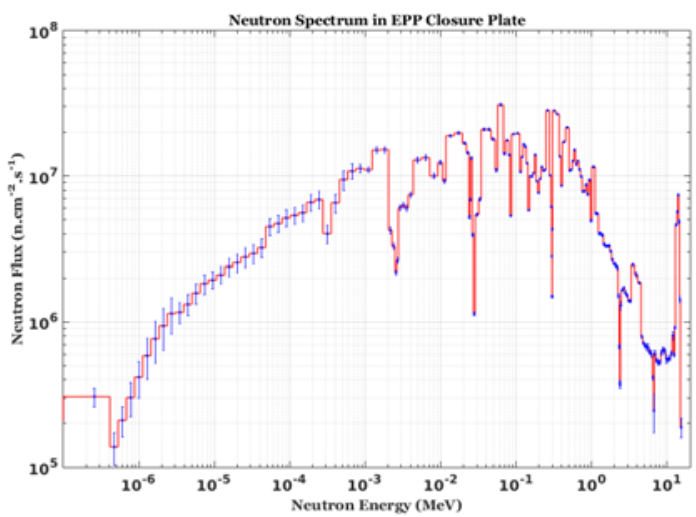

Figure 9. Neutron Spectrum in VITAMIN-J Energy Structure (175 groups) at the EPP Closure Plate $( \pm 2 \sigma)$ by MCNP-5.

Table 3. Comparison of neutron flux obtained at Closure Plate (CP) and Closure Flange (CF) by analog simulations with TRIPOLI-4 ${ }^{\circledR}$ and MCNP-5, based on the "dgepp-0-wavs" model.

\begin{tabular}{|c|c|c|c|c|}
\hline \multirow{2}{*}{ FENDL-2.1 } & \multicolumn{2}{|c|}{ Flux at CP } & \multicolumn{2}{c|}{ Flux at CF } \\
\cline { 2 - 5 } & $\mathbf{n . c ^ { - 2 }} \cdot \mathbf{s}^{-1}$ & $\sigma \%$ & $\mathbf{n . c m}^{-2} \cdot \mathbf{s}^{-1}$ & $\sigma \%$ \\
\hline TRIPOLI-4.10 & $8,14 \cdot 10^{9}$ & $2,0 \%$ & $6,66 \cdot 10^{9}$ & $3,0 \%$ \\
\hline MCNP-5.1.60 & $8,24 \cdot 10^{9}$ & $1,6 \%$ & $6,70 \cdot 10^{9}$ & $2,4 \%$ \\
\hline (T4-M5)/T4 \% & $-1,3 \%$ & $2,5 \%$ & $-0,6 \%$ & $3,8 \%$ \\
\hline
\end{tabular}




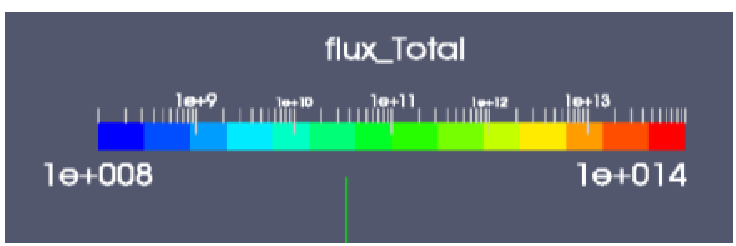

dgepp-0-wavs

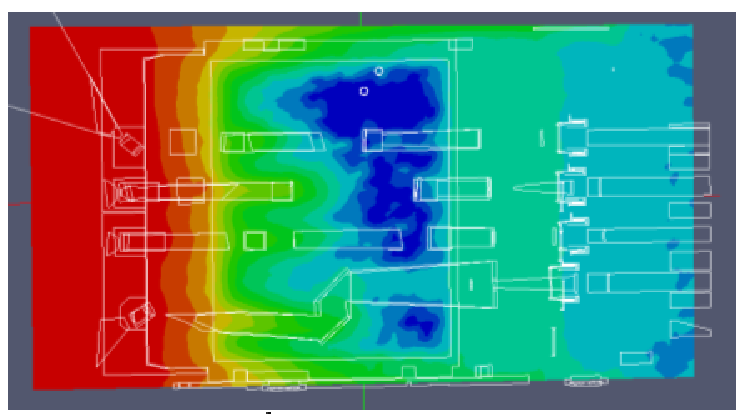

dgepp-1-wavs

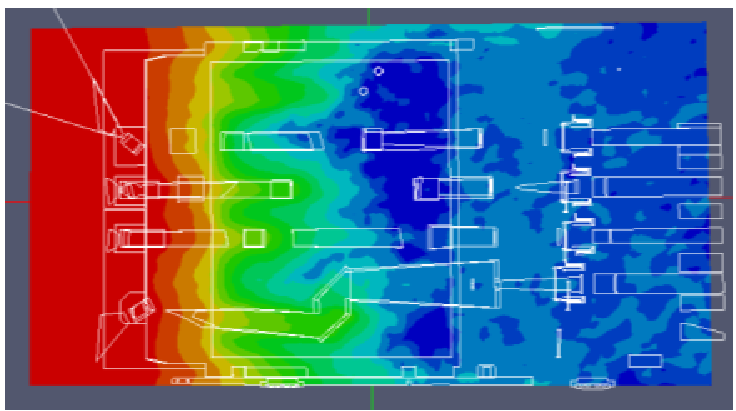

Figure 10. Neutron Flux maps obtained through the EPP (dgepp-0-wavs and dgepp-1-wavs models) by MCNP-5.

Table 4 summarizes the relative neutron flux results obtained for the different benchmark cases previously defined. Neutron flux maps obtained in the EPP area are depicted in Figure 10. These results were obtained with MCNP-5, using variance reduction techniques (based on the Weight Windows maps defined in section §2.3).

Table 4. Neutron flux results (in relative units) obtained at the closure plate for the different cases under study $(\sigma \sim 2 \%)$.

\begin{tabular}{|c|c|c|}
\hline & dgepp-0 & dgepp-1 \\
\hline baseline & 4 & 1 \\
\hline wavs & 10 & 1,1 \\
\hline
\end{tabular}

In figure 10, the dark blue regions inside the EPP can be explained by the strong neutron absorption in the boron carbide shielding modules. The shielding options adopted in dgepp-1 model (i.e. neutron labyrinths and neutron stoppers) are efficient in decreasing the neutron flux levels at the CP: a reduction factor of $\sim 10$ can be obtained in the "wavs" case (see Figure 10). However this factor is only $\sim 4$ for the "baseline" cases. This non-linearity could be explained by the existence of cross-talk phenomenon between the various streaming paths: from the intervessel gap into the optical path of the Divertor view for instance. This assumption should be verified: it could be further analysed using tally-tagging features.

\subsection{Sensitivity to Nuclear Data Library}

A sensitivity study is conducted to assess the impact of different nuclear data libraries on the results. Three libraries are used: FENDL-2.1, JEFF-3.1.1 [11], ENDFBVII.0. Results of neutron flux are reported in Table 5.

Table 5. Neutron Flux obtained at the EPP Closure Plate, results of TRIPOLI-4® simulations based on different nuclear data libraries.

\begin{tabular}{|c|c|c|}
\hline & $\Phi\left(\mathrm{n} \cdot \mathrm{cm}^{-2} \cdot \mathrm{s}^{-1}\right) \pm 1 \sigma$ & Rel. Diff. (\%) \\
\hline FENDL-2.1 & $8.14 \cdot 10^{9} \pm 2 \%$ & - \\
\hline JEFF-3.1.1 & $7.92 \cdot 10^{9} \pm 2 \%$ & $-2.7 \%$ \\
\hline ENDFB-VII.0 & $7.86 \cdot 10^{9} \pm 2 \%$ & $-3.3 \%$ \\
\hline
\end{tabular}

In terms of neutron shielding and streaming through the Equatorial Port Plug, no significant differences have been found between ENDFB-VII.0, JEFF-3.1.1 and FENDL2.1 .

\section{Conclusion and Perspectives}

A C-lite integrated neutronic model of ITER is developed for the Monte-Carlo code TRIPOLI-4 $\AA$. This model is equivalent to the $\mathrm{C}$-lite version 1 model officially released by ITER Organization for MCNP-5. This equivalence is verified by cross-checking the geometries and by doing the present benchmarking study.

It is noteworthy to mention that the newly developed model is originally produced for local shielding analysis, in the frame of design studies that are conducted for the WAVS diagnostics in the Equatorial Port Plugs. The scope of the benchmarking between TRIPOLI-4 ${ }^{\circledR}$ and MCNP-5 is thus limited to the assessment of neutron flux levels at the closure plate of the EPP. The neutroninduced activation of the closure plate (with subsequent decay-gamma emissions) is one of the most important contributions to the shutdown dose rate in the Interspace, where personnel access for maintenance is foreseen. The assessment of radiation levels behind the Equatorial Port Plugs is therefore a major concern for the Project.

The TRIPOLI-4® model has kept the same flexibility as the original MCNP-5 model to allow easy integration of detailed components when it is needed. Various DGEPP configurations were integrated in order to investigate shielding options. These integrations are based on CAD models and rely on MCAM, a CAD-toMonte-Carlo geometry conversion tool.

Based on the analysis of the various cases investigated, a few conclusions can be drawn: firstly, there is a significant neutron leakage in the gaps between the Vacuum Vessel and the Port Plug, and in the interDSM gaps. These streaming paths can be effectively shielded by the use of neutron labyrinths and neutron stoppers (absorbers). Secondly, the existence of cross-talk phenomenon between the various streaming paths makes it difficult to do separate-effect analysis, for instance by considering the impact of only one diagnostics among the others. For this reason, it is preferable to consider the problem as a whole, with all diagnostics included so far.

These preliminary results constitute a first step before the calculation of the shutdown dose rates in the 
Interspace. Shutdown dose rate calculations will be performed in the near future on the newly developed model. To be able to perform such calculations, the authors will rely on the on-going implementation of the so-called "rigorous-two-step" methodology [12] into TRIPOLI-4® [13]. Future work should also consider the optimization of the variance reduction scheme in TRIPOLI-4®, necessary to obtain reasonable statistical accuracy in the neutron flux meshes.

Finally, one of the main interests in developing alternative models for ITER is to provide independent validation of the results by code-to-code comparisons, thereby strengthening the confidence in Monte-Carlo simulations. This is especially important when variance reduction techniques are employed.

\section{Acknowledgments}

Part of this study was done with the financial support from Fusion for Energy in the frame of the contract F4EFPA-407. The views and opinions expressed herein do not necessarily reflect those of Fusion for Energy nor those of the ITER Organization.

\section{References}

1. S. Salasca et al., "The ITER Equatorial Visible/InfraRed Wide Angle Viewing System: Status of design and R\&D”, Fusion Engineering and Design, March 2015 .

2. R. Juárez, R. Pampin, B. Levesy, F. Moro, A. Suarez, J. Sanz, "Shutdown dose rates at ITER equatorial ports considering radiation cross-talk from torus cryopump lower port", Fusion Eng. Des., 100 (2015), pp. 501-506.

3. J.-C. Trama, E. Brun, F. Damian, C. Diop, E. Dumonteil, F.-X. Hugot, "TRIPOLI-4® version 9 Shielding for the fusion community: overview, relevant bench-marking for fusion and licencing policy”, ISFNT-11, Barcelona, Spain, September 1620, 2013.

4. X-5 Monte Carlo Team, "MCNP - A General Monte Carlo N-Particle Transport Code Version 5", LA-CP03-0245, Los Alamos National Laboratory, 2005.

5. D. Lopez Al-dama and A. Trkov, " FENDL-2.1: update of an evaluated nuclear data library for fusion applications Report INDC(NDS)-46 (Vienna: IAEA)", 2004.

6. SpaceClaim CAD Software: www.spaceclaim.com.

7. $\mathrm{Y} . \mathrm{Wu}$, "CAD-based interface programs for fusion neutron transport simulation FDS Team", Fusion Engineering and Design. vol. 84 (2009) pp. 1987 1992.
8. F.-X. Hugot and Y.-K. Lee, "A New Prototype Display Tool for the Monte-Carlo Particle Transport Code TRIPOLI-4®", Progress in Nuclear Science and Technology, Vol 2, pp.851-854, 2011.

9. J.-P. Both, J.-C. Nimal, T. Vergnaud, "Automated Importance Generation and Biasing Techniques for Monte-Carlo Shielding Techniques by the TRIPOLI3 Code", Progress in Nuclear Energy, Vol 24, pp.273-281, 1990.

10. O. Petit, Y.-K. Lee, C.M. Diop, "Variance Reduction Adjustment in Monte-Carlo TRIPOLI-4® Neutron Gamma Coupled Calculations", Progress in Nuclear Science and Technology, Vol 4, pp.408-412, 2014.

11. A. Santamarina et al., "The JEFF-3.1.1 nuclear data library, JEFF report 22, validation results from JEF2.2 to JEFF-3.1.1”, vol. 6807. OECD, NEA, 2009.

12. Y. Chen, U. Fischer, "Rigorous MCNP Based Shutdown Dose Rate Calculations: Computational Scheme, Verification Calculations and Application to ITER”, Fusion Engineering and Design, Vol. 63-64, (2002).

13. F. Malouch et al., "Recent developments in the TRIPOLI-4® Monte-Carlo code for fusion applications", in proceedings of SOFT 2016, Prague, Czech Republic. 